\section{Induced current magnetic resonance-electrical impedance tomography}

To cite this article: Levent Özparlak and Y Ziya der 2005 Physiol. Meas. 26 S289

View the article online for updates and enhancements.

\section{Related content}

Algebraic reconstruction for 3D MREIT

Y Ziya Ider and Serkan Onart

- Experimental results for MR-EIT

Özlem Birgül, B Murat Eyübolu and Y Ziya Ider

Equipotential projection for MR-EIT

Mahir S Özdemir, B Murat Eyübolu and Orçun Özbek

\section{Recent citations}

$$
\begin{aligned}
& \text { - } \frac{\text { bSSFP phase correction and its use in }}{\text { magnetic resonance electrical properties }} \\
& \frac{\text { tomography }}{\text { Safa Ozdemir and Yusuf Ziya Ider }} \\
& \text { - Induced Current Magnetic Resonance } \\
& \frac{\text { Electrical Conductivity Imaging With }}{\text { Oscillating Gradients }} \\
& \text { Hasan H. Eroglu et al } \\
& \text { - Electric properties tomography: } \\
& \text { Biochemical, physical and technical } \\
& \underline{\text { background, evaluation and clinical }} \\
& \frac{\text { applications }}{\text { Ulrich Katscher and Cornelius A.T. van }} \\
& \text { den Berg }
\end{aligned}
$$




\title{
Induced current magnetic resonance-electrical impedance tomography
}

\author{
Levent Özparlak and Y Ziya İder \\ Department of Electrical and Electronics Engineering, Bilkent University, Ankara, Turkey \\ E-mail: ozparlak@ee.bilkent.edu.tr and ider@ee.bilkent.edu.tr
}

Received 1 September 2004, accepted for publication 10 December 2004 Published 29 March 2005

Online at stacks.iop.org/PM/26/S289

\begin{abstract}
Magnetic resonance-electrical impedance tomography (MR-EIT) is a conductivity imaging method based on injecting currents into the object. In this study, a new MR-EIT method, whereby currents are induced inside the object by using external coils, is proposed. This new method is called induced current magnetic resonance-electrical impedance tomography. In induced current MREIT surface electrodes are not used and thereby artifacts due to electrodes are eliminated. The reconstruction algorithm is based on the measurement of only one component of the secondary magnetic flux density. The algorithm is an iterative one, is $3 \mathrm{D}$ and is based on the solution of a linear matrix equation at each iteration. For the measurement of secondary magnetic flux density, a pulse sequence to be used in the MRI system is proposed. Numerical simulations are performed to test the algorithm for both noise-free and noisy cases. The singular value behavior of the matrix is monitored and it is observed that at least two current induction profiles improve the images significantly. It is shown that induced current MR-EIT can be used to reconstruct absolute conductivity images without the need for any additional peripheral voltage measurement.
\end{abstract}

Keywords: electrical impedance tomography (EIT), MR-EIT, current density imaging (CDI), induced current MR-EIT

\section{Introduction}

Magnetic resonance-electrical impedance tomography (MR-EIT) methods proposed previously (Birgül and İder 1995, İder and Birgül 1998, Birgül et al 2003, Seo et al 2003a, 2003b, Kwon et al 2002, Khang et al 2002, Oh et al 2003, İder et al 2003, Ider and Onart 2004) are based on injecting current into the object to be imaged. These injected current MR-EIT methods suffer from problems caused by the current injection electrodes such as susceptibility artifacts and denser currents near the electrodes. To circumvent these problems, in this study, 
induced current MR-EIT is proposed. In this technique, similar to induced-current EIT (Gençer et al 1994), external coils are used to expose the object to an ac primary magnetic field which then generates eddy currents inside the object. These eddy currents create a new, secondary, magnetic flux density which can be measured by MRI. This secondary magnetic flux density contains the information necessary to reconstruct internal conductivity distribution.

MR-EIT techniques fall into two categories, those utilizing all three components of the secondary magnetic flux density and those utilizing only $B_{z}$, where $z$ is the direction of the main dc magnetic field of the MRI system. The former class of algorithms has the disadvantage that the object to be imaged has to be rotated in the MRI gantry because only one component of the secondary magnetic flux density can be measured by MRI at one time. Therefore in the induced current MR-EIT method proposed in this study a reconstruction algorithm based on $B_{z}$ only is utilized.

There are several MR-EIT reconstruction techniques based on $B_{z}$ only. Birgül and İder (1995), İder and Birgül (1998) and Birgül et al (2003) use an iterative sensitivity matrix method which utilizes the linearized relation between perturbation in $B_{z}$ and perturbation in conductivity. Seo et al (2003a) use the gradient of $B_{z}$, i.e. $\nabla B_{z}$, to reconstruct internal current density distribution in axially symmetric cylindrical sections, after which they use a $J$-substitution algorithm (Kwon et al 2002, Khang et al 2002) to reconstruct conductivity. Seo et al (2003b) and Oh et al (2003), have developed iterative algorithms which utilize the Laplacian of $B_{z}$, i.e. $\nabla^{2} B_{z}$. In these iterative algorithms, at each iteration the gradient of conductivity is found first. Seo et al (2003b) integrate this gradient on Cartesian lines to reconstruct conductivity. Oh et al (2003), on the other hand, use a layer potential technique to reconstruct conductivity from its gradient. Later, İder and Onart (İder et al 2003, İder and Onart 2004) proposed several reconstruction algorithms for both current density imaging and conductivity imaging by using only one component of magnetic flux density. In this study the methods of İder and Onart are adopted for induced current MR-EIT.

In summary, a new technique for conductivity imaging, induced current MR-EIT, is proposed in this study. The reconstruction algorithm is based on the measurement of only one component of the secondary magnetic flux density. The algorithm is an iterative one, is 3D and is based on the solution of a linear matrix equation at each iteration. Numerical simulations are performed to test the algorithm for both noise-free and noisy cases.

\section{Formulation of the forward problem}

Let $\Omega$ be a bounded and electrically conductive domain in $R^{3}$ with boundary $\Gamma$. Conductivity and resistivity distributions inside $\Omega$ are $\sigma$ and $\rho$, respectively, both assumed to be positive. By applying a low frequency magnetic flux density inside $\Omega$, a quasi-static current density distribution can be generated in $\Omega$. This distribution will be a function of conductivity distribution inside the object.

Maxwell's equations for a sinusoidally varying electromagnetic field in a linear, nonmagnetic, isotropic and conductive medium are as follows:

$$
\begin{aligned}
& \nabla \times \mathbf{E}=-\mathrm{j} \omega \mathbf{B} \\
& \nabla \times \mathbf{B}=\mu_{0}(\sigma+\mathrm{j} \omega \epsilon) \mathbf{E} \\
& \nabla \cdot \mathbf{E}=\frac{\rho_{v}}{\epsilon} \\
& \nabla \cdot \mathbf{B}=0
\end{aligned}
$$


with the continuity equation

$$
\nabla \cdot \mathbf{J}=-\mathrm{j} \omega \rho_{v}
$$

where $\rho_{v}$ is the charge density and $\mathbf{D}$ and $\mathbf{J}$ are related to $\mathbf{E}$ by

$$
\mathbf{J}=(\sigma+\mathrm{j} \omega \epsilon) \mathbf{E},
$$

and

$$
\mathbf{D}=\epsilon \mathbf{E} .
$$

Additionally, the relations between magnetic vector potential, A, magnetic flux density, $\mathbf{B}$, and electric field, $\mathbf{E}$, are

$$
\begin{aligned}
& \mathbf{B}=\nabla \times \mathbf{A} \\
& \mathbf{E}=-\nabla \Phi-\mathrm{j} \omega \mathbf{A}
\end{aligned}
$$

where $\Phi$ is the potential distribution in $\Omega$.

Derivation of the forward problem starts from the continuity equation. Since there is no charge density in the object, the continuity equation can be rewritten as

$$
\nabla \cdot((\sigma+\mathrm{j} \omega \epsilon) \mathbf{E})=0 .
$$

If we combine equations (9) and (10), we get

$$
\nabla \cdot((\sigma+\mathrm{j} \omega \epsilon) \nabla \Phi)=-\mathrm{j} \omega \nabla \cdot((\sigma+\mathrm{j} \omega \epsilon) \mathbf{A}) .
$$

By using the property $\nabla \cdot(f \mathbf{U})=f \nabla \cdot \mathbf{U}+\nabla f \cdot \mathbf{U}$, the above equation becomes

$$
\nabla \cdot((\sigma+\mathrm{j} \omega \epsilon) \nabla \Phi)=-\mathrm{j} \omega((\sigma+\mathrm{j} \omega \epsilon) \nabla \cdot \mathbf{A}+\nabla(\sigma+\mathrm{j} \omega \epsilon) \cdot \mathbf{A}) .
$$

In equation (8), the curl of $\mathbf{A}$ is defined as magnetic flux density. However, divergence of $\mathbf{A}$ must also be defined in order to specify $\mathbf{A}$ completely. Since $\mathbf{A}$ is an auxiliary function, we are free to define divergence of $\mathbf{A}$. Coulomb's gauge, which adopts $\nabla \cdot \mathbf{A}=0$, is a good selection (Gençer et al 1994). Then, the formulation becomes

$$
\nabla \cdot((\sigma+\mathrm{j} \omega \epsilon) \nabla \Phi)=-\mathrm{j} \omega \nabla(\sigma+\mathrm{j} \omega \epsilon) \cdot \mathbf{A} .
$$

Due to the fact that we work at low frequency, it is possible to make the assumption that $\omega \epsilon \ll \sigma$ (Gençer et al 1994), and therefore equations (2), (6) and (13) reduce to

$$
\begin{aligned}
& \nabla \times \mathbf{B}=\mu_{0} \mathbf{J} \\
& \mathbf{J}=\sigma \mathbf{E} \\
& \nabla \cdot(\sigma \nabla \Phi)=-\mathrm{j} \omega \mathbf{A} \cdot \nabla \sigma .
\end{aligned}
$$

Since there will be no current flow outside the object, the normal component of the current density on $\Gamma$ will be equal to zero. By using this fact, the boundary condition of the above partial differential equation becomes:

$$
\frac{\partial \Phi}{\partial n}=-\mathbf{j} \omega \mathbf{A} \cdot \mathbf{n}
$$

where $\mathbf{n}$ is the outward normal vector.

Another assumption used is that the secondary magnetic vector potential, $\mathbf{A}_{s}$, due to eddy currents is much smaller than the primary magnetic vector potential, $\mathbf{A}_{p}$, generated by the external induction coil (Gençer et al 1994). If we take the phase of the ac current in the external induction coil as the reference phase (zero phase), then $\mathbf{A}_{p}$ is purely real for our case, 


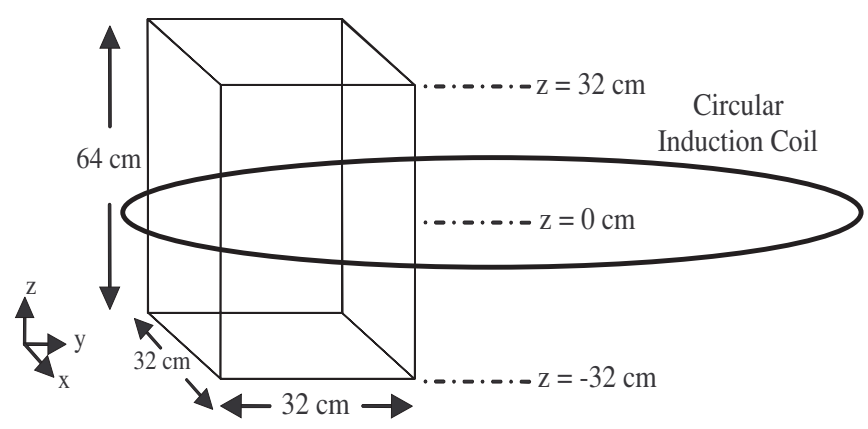

(a)

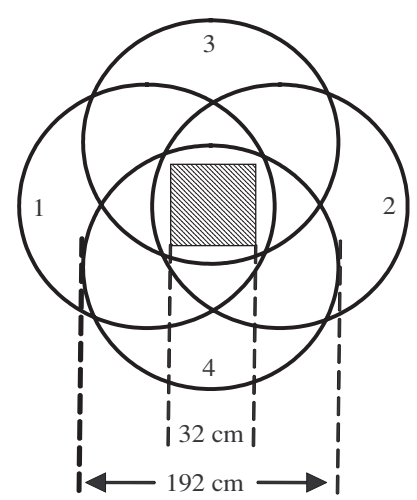

(b)

Figure 1. (a) Cuboid shaped simulation phantom and coil placement. (b) Top view of coil placements and simulation phantom.

i.e. low frequency quasi-static condition. Therefore equations (16) and (17) can be rewritten as

$$
\nabla \cdot(\sigma \nabla \Phi)=-\mathrm{j} \omega \mathbf{A}_{p} \cdot \nabla \sigma
$$

and

$$
\frac{\partial \Phi}{\partial n}=-\mathrm{j} \omega \mathbf{A}_{p} \cdot \mathbf{n}
$$

Note that since conductivity is a real positive quantity $\Phi$ must be purely imaginary. Under the same assumption equation (9) becomes

$$
\mathbf{E}=-\nabla \Phi-\mathrm{j} \omega \mathbf{A}_{p},
$$

and consequently it can be seen from equations (20) and (15) that $\mathbf{E}$ and $\mathbf{J}$ are also purely imaginary vector quantities.

Secondary magnetic flux density $\mathbf{B}_{s}$, due to $\mathbf{J}$, is given by the Biot-Savart integral

$$
\mathbf{B}_{s}(\mathbf{r})=\frac{\mu_{0}}{4 \pi} \int_{\Omega} \mathbf{J}\left(\mathbf{r}^{\prime}\right) \times \frac{\mathbf{r}-\mathbf{r}^{\prime}}{\left|\mathbf{r}-\mathbf{r}^{\prime}\right|^{3}} \mathrm{~d} v^{\prime}
$$

where $\mathbf{r}$ and $\mathbf{r}^{\prime}$ are field and source vectors defined in $\Omega$. Primary magnetic flux density $\mathbf{B}_{p}$, due to the external induction coil (assumed to be circular and lying in the $x y$-plane) is given by another Biot-Savart integral

$$
\mathbf{B}_{p}(\mathbf{r})=\frac{\mu_{0} I_{\text {coil }} a}{4 \pi} \int_{C} \mathbf{a}_{\phi} \times \frac{\mathbf{r}-\mathbf{r}^{\prime}}{\left|\mathbf{r}-\mathbf{r}^{\prime}\right|^{3}} \mathrm{~d} l^{\prime}
$$

where $\mathbf{r}$ is the field vector in $\Omega, \mathbf{r}^{\prime}$ is the source vector on the coil $I_{\text {coil }}$ is the current flowing in the coil, $C$ is the contour of the coil, $a$ is the radius of the coil, $\mathrm{d} l^{\prime}$ is the incremental length of a current element on the coil and $\mathbf{a}_{\phi}$ is the unit vector of the incremental current element. $\mathbf{B}_{s}$ is purely imaginary and $\mathbf{B}_{p}$ is purely real.

The finite element method (FEM) is used to solve equation (18) for the boundary condition in equation (19). In figure 1(a), the object, which is a cuboid, used in the simulation studies is shown. The object is divided into small cubic elements using a regular Cartesian mesh. In figure 2(a) the Cartesian mesh of cubic elements for an $x y$-slice of the cuboid object is illustrated. It is assumed that each cubic element has constant conductivity inside. Each cubic element is divided into six tetrahedral elements (Silvester and Ferrari 1996). 


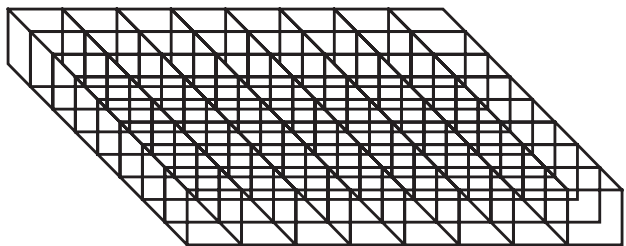

(a)

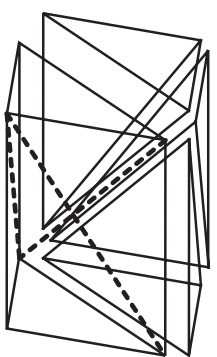

(b)

Figure 2. (a) A Cartesian mesh of cubic elements for an $x y$-slice of the cuboid object. (b) Six tetrahedra forming a cubic element.

In figure 2(b), the six tetrahedra of a cubic element are shown. $\Phi$ is approximated by a first-order polynomial inside a tetrahedral element. In addition, $\mathbf{A}_{p}$ is assumed to be constant inside a tetrahedral element. $\mathbf{E}$ and $\mathbf{J}$ are also constant since $\mathbf{A}_{p}$ is constant inside the element and $\Phi$ is planar. To find $\mathbf{E}$ and $\mathbf{J}$ in a cubic element, the average of the corresponding values for the six tetrahedral elements making up the same cubic element is used and new $\mathbf{E}$ and $\mathbf{J}$ are assumed to be at the centers of cubes.

The FEM matrix generated for the solution of $\Phi$ is singular and it is necessary to assign a reference voltage value to any one of the mesh nodes. The value of the reference voltage is irrelevant because in finding $\mathbf{E}$ and $\mathbf{J}$ the gradient of $\Phi$ is used. The size of the FEM matrix is very large. In our simulation experiments, its size is $32 \times 32 \times 64 \times 6$ by $32 \times 32 \times$ $64 \times 6$ as explained in section 6 . To lower the computational cost of the FEM solution, the preconditioned conjugate gradients method (PCG of Matlab) is used and sparsity of the FEM matrix is taken into consideration.

Secondary magnetic flux density is calculated by discretizing the Biot-Savart integral. During the calculation, it is assumed that there exist current sources located at the center of gravity of each tetrahedron, and the field points are selected as the center of each cubic element.

\section{Formulation of the inverse problem}

Magnetic flux density has real and imaginary parts. Due to the assumptions explained in section 2, equation (1) becomes

$$
\nabla \times\left(\mathbf{E}_{R}+\mathrm{j} \mathbf{E}_{I}\right)=-\mathrm{j} \omega\left(\mathbf{B}_{p}+\mathrm{j} \mathbf{B}_{s}\right) .
$$

The imaginary part of equation $(23)$ is

$$
\nabla \times \mathbf{E}_{I}=-\omega \mathbf{B}_{p}
$$

By using the inverse of equation (15), i.e. $\mathbf{E}=\rho \mathbf{J}$, we get

$$
\nabla \times \rho \mathbf{J}_{I}=-\omega \mathbf{B}_{p}
$$

where $\mathbf{J}_{I}$ itself is the eddy current density since $\mathbf{J}$ is purely imaginary. An equivalent form of equation (25) is

$$
\nabla \rho \times \mathbf{J}_{I}+\rho \nabla \times \mathbf{J}_{I}=-\omega \mathbf{B}_{p} .
$$


The imaginary part of the current density distribution will be directly related to $\mathbf{B}_{s}$ by using equation (14), and the curl of $\mathbf{J}_{I}$ can be expressed as

$$
\nabla \times \mathbf{J}_{I}=\nabla \times \frac{\nabla \times \mathbf{B}_{s}}{\mu_{0}}=\frac{1}{\mu_{0}}\left(\nabla\left(\nabla \cdot \mathbf{B}_{s}\right)-\nabla^{2} \mathbf{B}_{s}\right)=-\frac{\nabla^{2} \mathbf{B}_{s}}{\mu_{0}} .
$$

By inserting equation (27) into equation (26), we get

$$
\nabla \rho \times \mathbf{J}_{I}-\rho \frac{\nabla^{2} \mathbf{B}_{s}}{\mu_{0}}=-\omega \mathbf{B}_{p} .
$$

The third component of the above vectoral equation system is

$$
\frac{\partial \rho}{\partial x} J_{y}-\frac{\partial \rho}{\partial y} J_{x}-\rho \frac{\nabla^{2} B_{s z}}{\mu_{0}}=-\omega B_{p z}
$$

where $J_{x}$ and $J_{y}$ represent the $x$ and $y$ components of current density $\mathbf{J}_{I}$ respectively, and $B_{s z}$ and $B_{p z}$ are the $z$ components of the corresponding field quantities.

Assuming that $B_{s z}$ is measured and $B_{p z}$ is known, this equation is a partial differential equation for $\rho$ and it is the basis for the solution of the inverse problem. It is a nonlinear PDE because $J_{x}$ and $J_{y}$ depend on $\rho$. This equation can be solved iteratively to obtain the $\rho$ distribution on a $x y$-slice by $x y$-slice basis (İder et al 2003, İder and Onart 2004) as explained in the next section.

\section{Numerical solution of the inverse problem}

Equation (29) is discretized for any $x y$-slice by using finite differences. The discretization points are the centers of the cubic elements of the selected slice, say the $k$ th slice. Let us assume that the Cartesian mesh contains $N$ cubic elements in the $x$ direction and $M$ cubic elements in the $y$ direction. Then, there exist $N M$ cubic elements in each slice. The resistivity values of these cubic elements are denoted by $\mathbf{R}$ which is an $N M \times 1$ vector. In inner regions of the slice, discretization is done with central difference formulation. On the edges and corners, backward or forward difference formulations are used where appropriate. For example, the discretized equation for the $(i, j)$ th cubic element, which is in the inner region, i.e. $2<i<N-1$ and $2<j<M-1$, is

$$
\begin{gathered}
\frac{\rho(i+1, j, k)-\rho(i-1, j, k)}{2 \Delta x} J_{y}(i, j, k)-\frac{\rho(i, j+1, k)-\rho(i, j-1, k)}{2 \Delta y} J_{x}(i, j, k) \\
-\rho(i, j, k) \frac{\left(\nabla^{2} B_{s z}\right)_{(i, j, k)}}{\mu_{0}}=-\omega B_{p z}(i, j, k)
\end{gathered}
$$

where the discretized version of the Laplacian operator for a scalar function $U$ for the $k$ th slice (not on the boundary) is

$$
\begin{aligned}
\left(\nabla^{2} U\right)_{(i, j, k)}= & (1 / 6)(U(i+1, j, k)+U(i-1, j, k)+U(i, j+1, k) \\
& +U(i, j-1, k)+U(i, j, k+1)+U(i, j, k-1)-6 U(i, j, k)) .
\end{aligned}
$$

There are $N M$ equations, one for each cubic element, and $N M$ resistivity unknowns. We rearrange and combine all the equations into a matrix form as

$$
\mathbf{S R}=\mathbf{b}
$$

where $\mathbf{S}$ is the $N M \times N M$ coefficient matrix, $\mathbf{R}$ is the $N M \times 1$ vector of unknowns and $\mathbf{b}$ is the $N M \times 1$ vector of $-\omega B_{p z}$.

It is possible to take more than one set of measurements with different coil placements. In figure 1(b), four circular coils which are centered at different locations are shown. We call 
the combination of the type of coil and where the coil is placed the current induction profile. If there are $K$ different current induction profiles, there will be $K$ different $J_{x}, J_{y}, \nabla^{2} B_{s z}$ and $B_{p z}$ distributions, and consequently there will be $K$ different $\mathbf{S}$ matrices and $\mathbf{b}$ vectors. We rename these as $J_{x \ell}, J_{y \ell}, \mathbf{S}_{\ell}$ and $\mathbf{b}_{\ell}$ where $\ell=1,2, \ldots, K$. We concatenate all $\mathbf{S}_{\ell}$ matrices and $\mathbf{b}_{\ell}$ vectors to form the combined system equation

$$
\Sigma \mathbf{R}=\beta
$$

where $\Sigma=\left[\mathbf{S}_{1}^{T} \mathbf{S}_{2}^{T} \cdots \mathbf{S}_{K}^{T}\right]^{T}$ is the combined system matrix and $\beta=\left[\mathbf{b}_{1}^{T} \mathbf{b}_{2}^{T} \cdots \mathbf{b}_{K}^{T}\right]^{T}$. Note that $\mathbf{R}$ is the vector of unknown resistivities of the $k$ th slice. We define $\boldsymbol{\Theta}$ as the vector of unknown resistivities of the whole $3 \mathrm{D}$ object formed by concatenating the $\mathbf{R}$ vectors of the slices.

Equation (33) is nonlinear in $\mathbf{R}$ because the $J_{x \ell}$ and $J_{y \ell}$ which appear in the $\mathbf{S}_{\ell}$ matrices are dependent on $\mathbf{R}$. Therefore, an iterative method for reconstruction is proposed. The steps of the iterative method can be defined as:

Step 1. Assume an initial $\Theta_{0}$ distribution for the 3D object to be imaged. Usually $\Theta_{0}$ is taken to be the uniform distribution. Set iteration number $i$ to 1 .

Step 2. Take $\Theta_{i}=\Theta_{i-1}$. Calculate $J_{x \ell}$ and $J_{y \ell}, \ell=1, \ldots, K$ for all slices simultaneously using the 3D FEM solver for $\Theta_{i}$.

Step 3. Calculate $\mathbf{b}_{\ell}, \ell=1, \ldots, K$ by using known $B_{p z}$ information for all current induction profiles, for all slices.

Step 4. Calculate the Laplacian of measured $B_{s z}$ for all profiles and construct $\mathbf{S}_{\ell}, \ell=1, \ldots, K$, for all slices.

Step 5. For each slice, concatenate $\mathbf{S}_{\ell}$ matrices and $\mathbf{b}_{\ell}$ vectors to obtain the $\Sigma$ matrix and $\beta$ vector of that slice.

Step 6. Solve equation (33) for each slice.

Step 7. Form a new $\boldsymbol{\Theta}_{i}$ from the $\mathbf{R}$ vectors of all slices and check for the stopping condition and stop if it is met. Else, increase $i$ by 1 and go to step 2 .

In step 6, equation (33) is solved in the least-squares sense by solving

$$
\Sigma^{T} \Sigma \mathbf{R}=\Sigma^{T} \beta
$$

As will be shown later, for the simulation experiments made in this study, $\Sigma^{T} \Sigma$ is non-singular, and LU factorization with partial pivoting is used for the solution of equation (34) (INV of Matlab).

The stopping condition in step 7 may be selected in many different ways. One way of selecting where to stop the iteration is to look at the relative $L^{2}$ norm error. Relative $L^{2}$ norm error is defined as

$$
L_{\text {rel.err }}^{2}=100 \frac{\left\|\Theta-\Theta_{\text {org }}\right\|_{L_{2}}}{\left\|\Theta_{\text {org }}\right\|_{L_{2}}}
$$

where $\Theta_{\text {org }}$ is the original resistivity distribution. Note that this error measure can only be used in simulations in which $\Theta_{\text {org }}$ is known, in order to observe the convergence properties of the algorithm.

\section{The proposed pulse sequence}

MRI techniques for measuring the magnetic flux density generated by a current density distribution are developed by investigators who aim at current density imaging (CDI). For the case of injected dc currents, Scott et al (1992) have used a spin echo pulse sequence which 


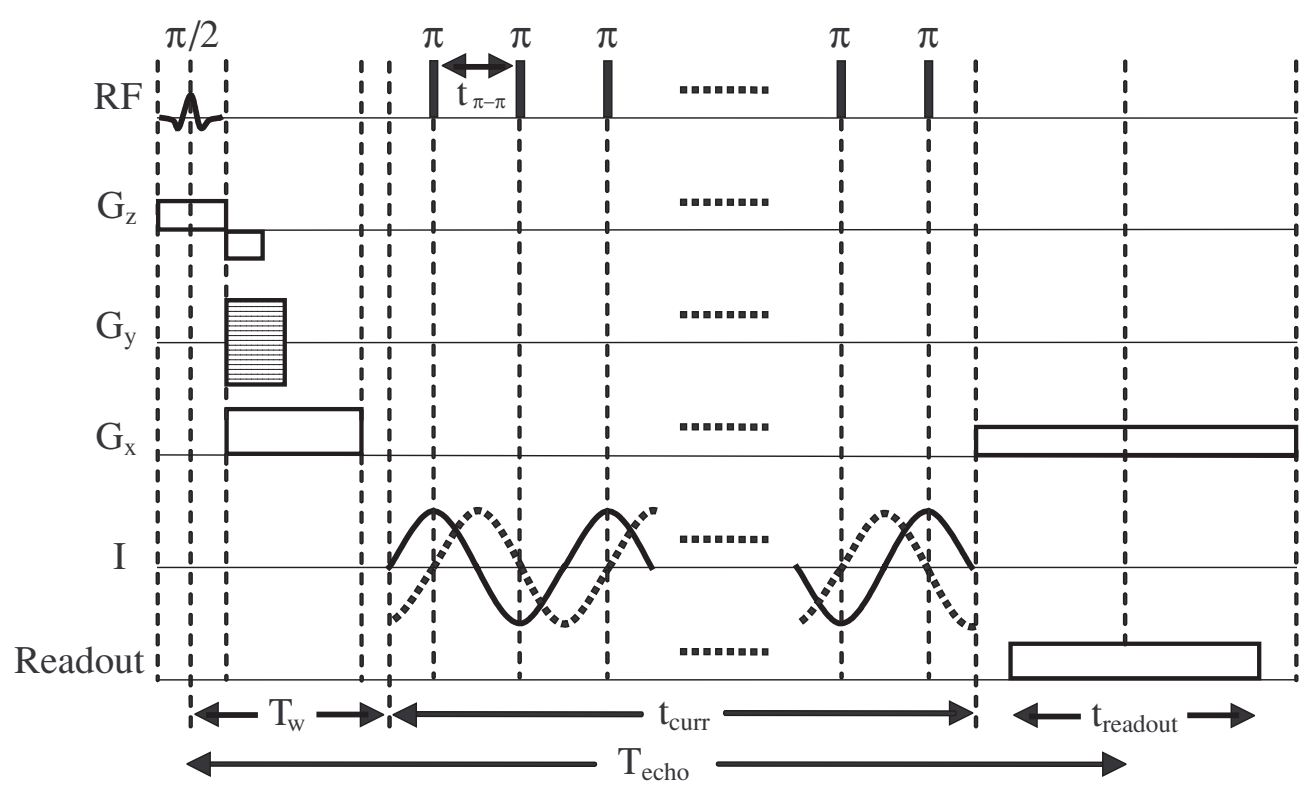

Figure 3. The proposed pulse sequence for induced current MR-EIT. I, shown by the solid line, is the current flowing through the excitation coil. $B_{p z}$ is in-phase with $I$, but $B_{s z}$ is out-of-phase with it as illustrated by the broken line on the same axis with $I$. RF line represents the RF pulses. $G_{x}, G_{y}$ and $G_{z}$ are the applied gradients in the $x, y$ and $z$ directions during the spin echo experiment, respectively. $t_{\text {curr }}, T_{\text {echo }}, t_{\text {readout }}, t_{\pi-\pi}, T_{w}$ are the duration of applied current, the echo time, the duration of readout, the time between successive $180^{\circ} \mathrm{RF}$ pulses and the time between the $90^{\circ} \mathrm{RF}$ pulse and the first $180^{\circ} \mathrm{RF}$ pulse respectively.

has a single $180^{\circ} \mathrm{RF}$ pulse. For the case of injected ac currents of $1 \mathrm{kHz}$ frequency, Mikac et al (2001) have developed a method which involves multiple $180^{\circ} \mathrm{RF}$ pulses. The MRI pulse sequence used by Mikac et al can be adapted to the particular case of induced ac currents used in this study, as shown in figure 3. During ac excitation, $180^{\circ}$ RF pulses are applied at the peaks of $B_{p z}$, and therefore phase accumulating due to $B_{p z}$ is zero for each half-cycle. However, since $B_{s z}$ and $B_{p z}$ are out-of-phase by $90^{\circ}, 180^{\circ} \mathrm{RF}$ pulses coincide with the zero crossings of $B_{s z}$. Therefore phases accumulating due to $B_{s z}$ at each half-cycle add up. Total phase accumulating during current application is therefore due only to $B_{s z}$ and is equal to

$$
\varphi_{S}(x, y, z)=(-1)^{N} \gamma\left\langle B_{s z}(x, y, z)\right\rangle t_{\text {curr }}
$$

where $N$ is the number of $180^{\circ}$ RF pulses, $\gamma$ is the gyro-magnetic ratio $(26.75 \times$ $\left.10^{7} \mathrm{rad} \mathrm{s}^{-1} \mathrm{~T}^{-1}\right), t_{\text {curr }}$ is the duration of the applied current $I$ and $\left\langle B_{s z}\right\rangle$ is the time average value of $B_{s z}$ during one positive half-cycle, that is, $(2 / \pi) B_{s z}^{\text {peak }}$ (peak during one positive half-cycle).

In order to obtain more sensitivity and also to eliminate the constant phase terms due to static magnetic field inhomogeneities and delays due to MRI hardware, the proposed pulse sequence is applied twice with the current $I$ reversed in the second application. The phase difference of the complex MR images obtained for the two applications is given by

$$
\varphi_{S 2}(x, y, z)=2(-1)^{N} \gamma B_{s z}^{\text {peak }}(x, y, z) \frac{2 t_{\text {curr }}}{\pi} .
$$

Since $t_{\pi-\pi}$ is much smaller than twice $T_{w}$, spin echoes do not occur between $180^{\circ} \mathrm{RF}$ pulses. Instead, a spin echo is only formed at the end, during $t_{\text {readout }}$, provided that $N$ is odd. To use similar parameters as used in Mikac et al (2001), we have chosen $T_{w}=6 \mathrm{ms,}$ 


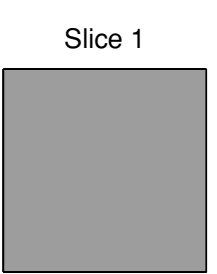

Slice 33

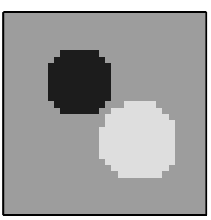

Slice 23

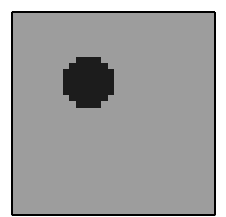

Slice 38

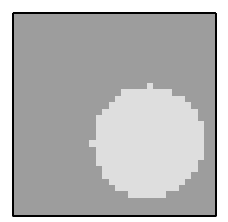

Slice 27

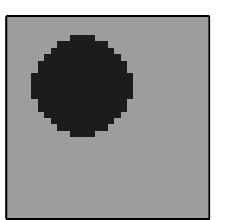

Slice 42

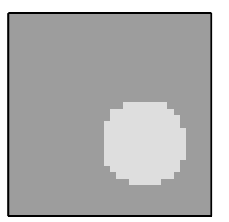

Slice 32

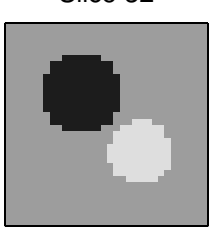

Slice 64

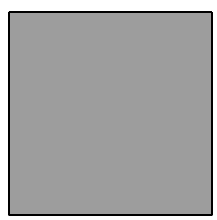

$\mathrm{S} / \mathrm{m}(\Omega-\mathrm{m})$

0.45 (2.22)

$0.40(2.50)$

0.35 (2.86)

0.30 (3.33)

$0.25(4.00)$

$0.20(5.00)$

0.15 (6.67)

$0.10(10.0)$

$\square_{0.05}(20.0)$

Figure 4. Original conductivity distributions used in the simulations for eight different slices of the first simulation phantom.

$t_{\text {curr }}=19.5 \mathrm{~ms}, N=39$ and $T_{\text {echo }}=31.5 \mathrm{~ms}$. With these parameters and maximum $B_{s z}^{\text {peak }}=$ $30 \mathrm{nT}$ as in the first simulation phantom (described in the next section), the phase accumulating due to $B_{s z}$ is $14.3^{\circ}$.

In the last section, various practical aspects of the proposed pulse sequence are discussed.

\section{Simulation results}

Computer simulations are made in order to assess the performance of the proposed induced current MR-EIT method. A cuboid shaped object with edge lengths $32 \mathrm{~cm} \times$ $32 \mathrm{~cm} \times 64 \mathrm{~cm}$ in the $x, y$ and $z$ directions, respectively, is used as the basis of our simulation phantoms (see figure 1(a)). This rectangular object is divided into $1 \mathrm{~cm} \times 1 \mathrm{~cm} \times 1 \mathrm{~cm}$ cubic elements using a regular Cartesian mesh. The $x y$-slices are numbered from 1 to 64 starting from the bottom of the object. For example, the 32 nd slice is the slab between $z=-1 \mathrm{~cm}$ and $z=0 \mathrm{~cm}$ planes. The first simulation phantom contains two spheres which are centered at different $x y$-slices and which have different conductivity values. One has a conductivity value of $0.4 \mathrm{~S} \mathrm{~m}^{-1}$, and the other has a conductivity value of $0.1 \mathrm{~S} \mathrm{~m}^{-1}$. Background conductivity is taken as $0.2 \mathrm{~S} \mathrm{~m}^{-1}$. In figure 4, conductivity distributions of eight selected slices of the first simulation phantom are given.

In figure 1(b), the external induction coils are illustrated with reference to the simulation object. Four external circular coils with diameters $192 \mathrm{~cm}$ are used to generate the primary magnetic flux density excitation. These coils are placed with centers at $(x, y, z)=$ $(-32,0,0),(32,0,0),(0,-32,0)$ and $(0,32,0)$, where quantities are in $\mathrm{cm}$. These coils are excited one at a time and it is assumed that $B_{s z}$ is measured for these four cases. For simulation purposes $B_{s z}$ is calculated to generate simulation data.

The reconstructed conductivity distribution for the first simulation phantom, for the fifth iteration, is given in figure 5. The initial uniform conductivity distribution is taken to be $0.05 \mathrm{~S} \mathrm{~m}^{-1}$. It is observed that boundaries and locations of the spheres are well reconstructed with high spatial resolution in all slices. In figure 6, results for the first five iterations are given for the 32nd slice (mid-slice). From the profile plots, the convergence characteristics of the algorithm can be observed. The relative $L^{2}$ norm errors for the first five iterations are $59.4 \%$, $14.6 \%, 13.3 \%, 13.3 \%$ and $13.3 \%$. 


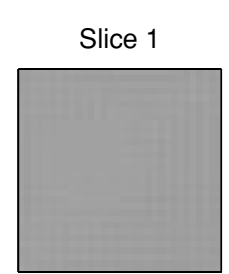

Slice 33

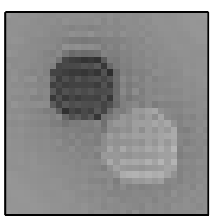

Slice 23

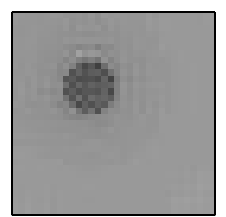

Slice 38

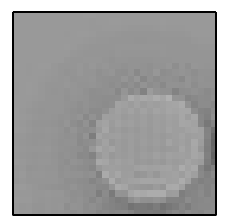

Slice 27

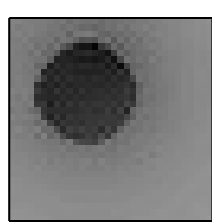

Slice 42

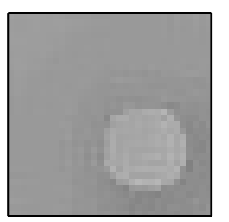

Slice 32

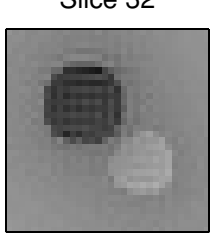

Slice 64

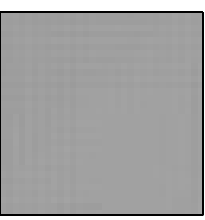

$\mathrm{S} / \mathrm{m}(\Omega-\mathrm{m})$

0.45 (2.22)

$0.40(2.50)$

$0.35(2.86)$

$0.30(3.33)$

$0.25(4.00)$

$0.20(5.00)$

$0.15(6.67)$

$0.10(10.0)$

$\sqcup_{0.05}(20.0)$

Figure 5. Fifth iteration reconstructed conductivity distributions for eight different slices of the first simulation phantom.
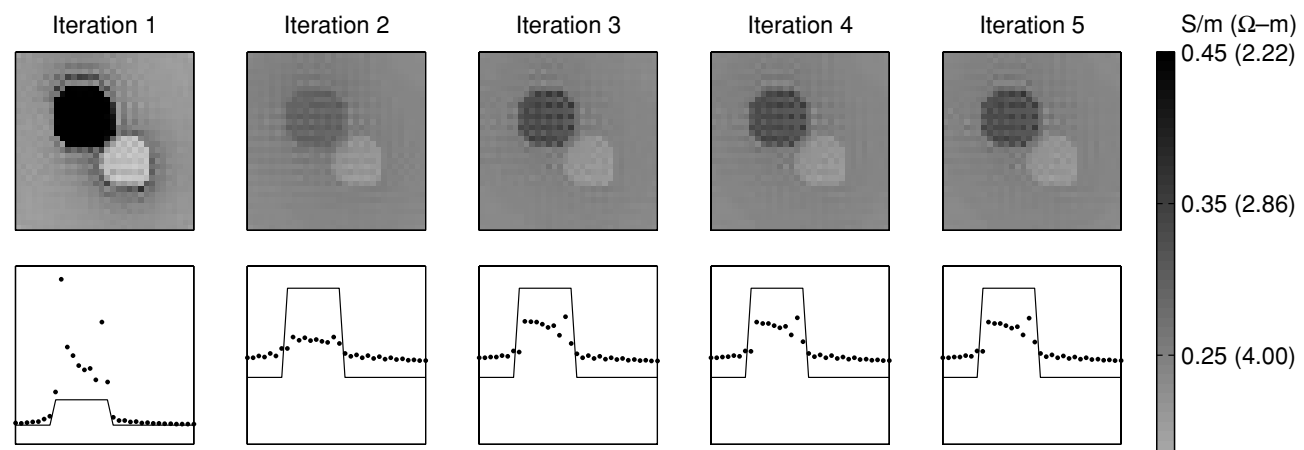

$0.35(2.86)$
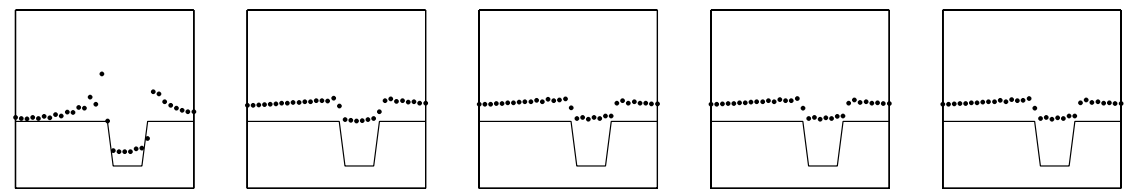

Figure 6. First five iteration images for slice 32 of the first simulation phantom and two different profile plots of the corresponding images. The first profile plot is for the horizontal line which passes through the center of the higher circular conductivity region. Similarly, the second profile plot is for the horizontal line which passes through the center of the lower circular conductivity region. In each profile plot, the original conductivity values are shown by solid lines and the reconstructed conductivity distributions are shown by dots. The scales are taken to be between 0.45 and 0.05 for the profile plots except for the first profile plot in the iteration 1 column for which the scale is between 1.45 and 0.05 .

In figure 7, some simulation results for the second simulation phantom containing a more complicated conductivity distribution are given. The second simulation phantom is a simple model of the human thorax, but its conductivity distribution does not have $z$-dependence. It contains less conductive lung $\left(0.181-0.061 \mathrm{~S} \mathrm{~m}^{-1}\right)$, bone $\left(0.143-0.055 \mathrm{~S} \mathrm{~m}^{-1}\right)$ and more conductive heart $\left(0.4-0.22 \mathrm{~S} \mathrm{~m}^{-1}\right)$ regions. In this phantom, as compared to the first simulation phantom, edges of the different conductivity regions are smoothly varying. Figure 7 contains four images, which are the original conductivity distribution of mid-slice and the reconstruction 


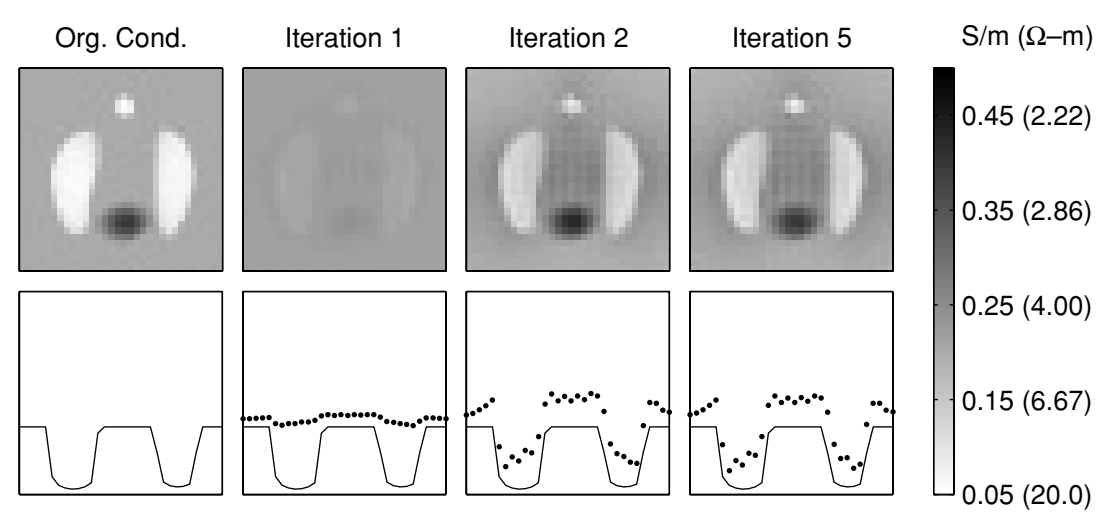

Figure 7. The original conductivity distribution, the reconstructed conductivity distributions for the first, second and fifth iterations for slice 32 and their profile plots for the second simulation phantom. The profile plots are for the mid-vertical lines. In each profile plot, the original conductivity values are shown by solid lines and the reconstructed conductivity distributions are shown by dots.
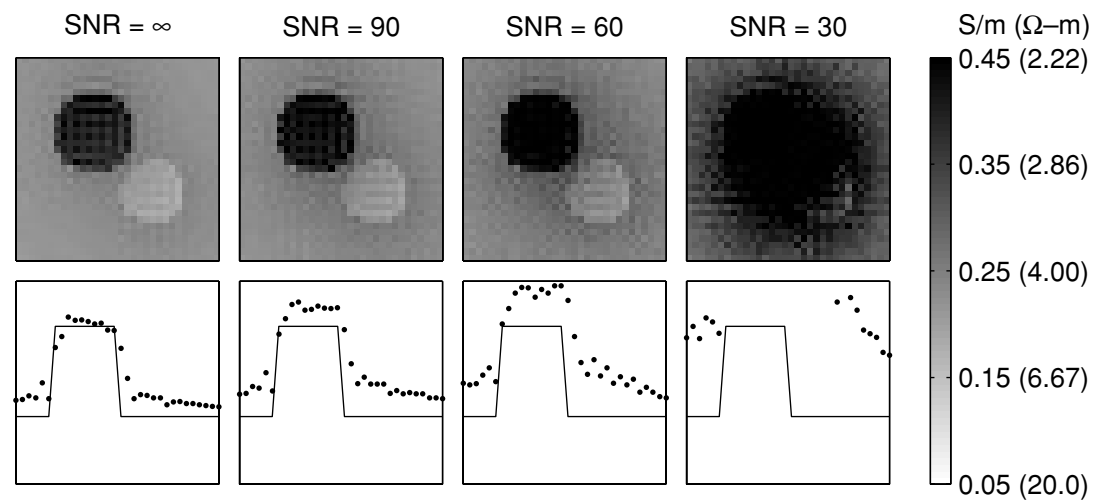

Figure 8. Reconstructed conductivity distributions for the first iteration for slice 32 of the first simulation phantom and their profile plots for MRI with system SNR $=\infty, 90,60$ and 30. The profile plots are for the mid-vertical lines. In each profile plot, the original conductivity values are shown by solid lines and the reconstructed conductivity distributions are shown by dots. The initial uniform conductivity distribution was taken to be $0.2 \mathrm{~S} \mathrm{~m}^{-1}$.

results for the first, second and fifth iterations. The initial uniform conductivity distribution is taken to be $0.2 \mathrm{~S} \mathrm{~m}^{-1}$. The relative $L^{2}$ norm errors for the first five iterations are $33.9 \%$, $25.6 \%, 25.1 \%, 25.2 \%$ and $25.2 \%$. If the simulation results and the relative $L^{2}$ norm errors of the first and fifth iterations are compared, a significant improvement in the conductivity image can be observed.

Simulations for noisy $B_{s z}$ data are also made to analyze the behavior of the algorithm against noise. The noise model in Scott et al (1992) is used as modified in Idder and Onart (2004). In this modified model, zero mean Gaussian noise with standard deviation of $\sigma_{s}=1 /\left(2 / \gamma t_{\text {curr }}\right.$ $\mathrm{SNR}$ ), where SNR is the signal-to-noise ratio of the MRI system, is added to $B_{s z}$. Simulation results for the first phantom for $t_{\text {curr }}=19.5 \mathrm{~ms}$ and $\mathrm{SNR}=\infty, 90,60$ and 30 are given in figure 8. The standard deviations for SNR $=30,60$ and 90 are $3.195 \mathrm{nT}, 1.598 \mathrm{nT}$ and $1.065 \mathrm{nT}$, respectively. The relative $L^{2}$ errors for $\mathrm{SNR}=\infty, 90,60$ and 30 are $12.1 \%$, $24.4 \%, 45.4 \%$ and $190.7 \%$, respectively. As also observed from figure 8, SNR values larger 


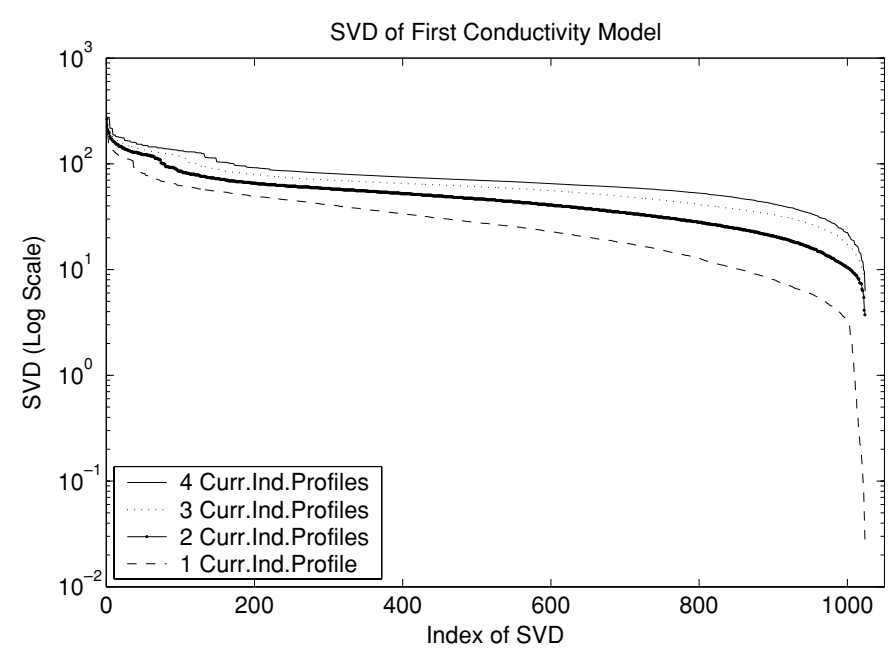

Figure 9. Singular values of the combined system matrix on a logarithmic scale. One current induction profile means that only coil 1 in figure 1 is excited. Two current induction profiles means that coils 1 and 2 are excited. Three current induction profiles means coils 1, 2 and 3 are excited. Four current induction profiles means that all four coils are excited. The vertical axis shows the normalized singular values.

than 60 are necessary for at least maintaining internal boundary information. It is known that much larger SNR values are practically achievable in real MRI systems. For body coils 200 SNR is possible (Schnell et al 2000). For head and other special coils higher values can be obtained.

Figure 9 shows the logarithmic singular value plots for the combined system matrix for the first iteration of slice 32 of the first simulation phantom, for different current induction profile combinations. The initial uniform conductivity distribution was taken to be $0.2 \mathrm{~S} \mathrm{~m}^{-1}$. For one current induction profile, the size of the system matrix is $1024 \times 1024$. For two, three and four current profiles, the size of the system matrix increases to $2048 \times 1024,3096 \times$ 1024 and $4192 \times 1024$, respectively. Whether one, two, three or four current induction profiles are used, it is observed that the rank of the system matrix is 1024. The condition number of the combined system matrix is $8218,71,53,44$ for one, two, three and four current induction profiles, respectively. The condition number is a measure of the ill-conditioning of the system matrix and indicates how tolerant the system is against measurement noise. In our case, it is apparent that use of more than one current profile improves the condition number significantly. Since the condition number for four current induction profiles is better than any of the other cases, all simulation results are obtained using four current induction profiles.

In figure 10, the first iteration reconstructed images for one, two, three and four current profiles are given. The initial uniform conductivity distribution was taken to be $0.2 \mathrm{~S} \mathrm{~m}^{-1}$. When one current induction profile is used, the combined system matrix is relatively more ill-conditioned and the reconstructed image is dissatisfactory. However, with two, three or four current induction profiles, increasingly more satisfactory conductivity images are obtained.

\section{Discussion and conclusions}

In injected current MR-EIT, the absolute conductivity distribution inside the object cannot be found. There is a need for an additional peripheral voltage measurement to find the 

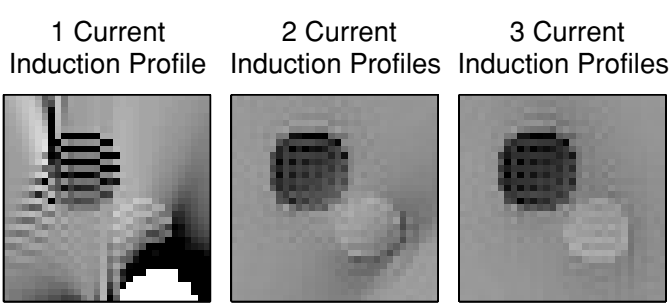

4 Current

Induction Profiles

$\mathrm{S} / \mathrm{m}(\Omega-\mathrm{m})$

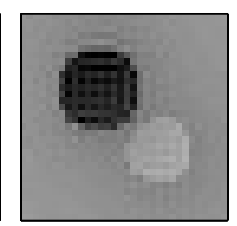

0.45 (2.22)

0.35 (2.86)

0.25 (4.00)

0.15 (6.67)

$0.05(20.0)$

Figure 10. Reconstructed conductivity distributions for the first iteration for slice 32 of the first simulation phantom, for the cases of one, two, three and four current induction profiles are used in the simulation.

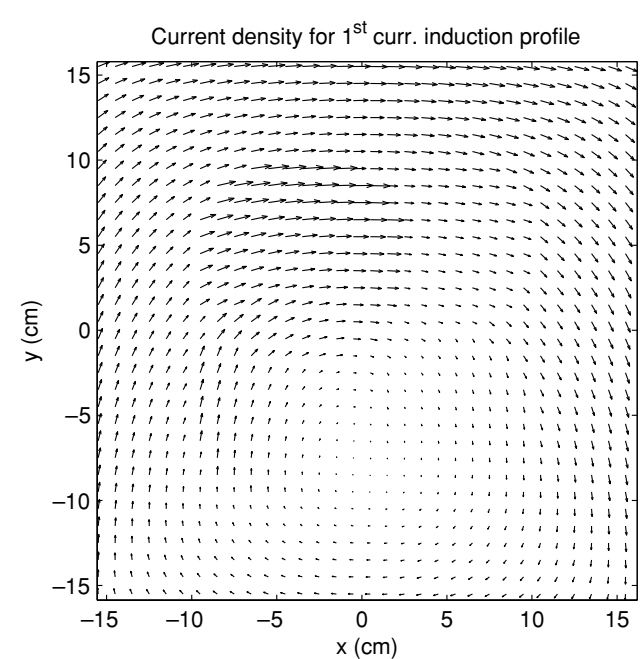

(a)

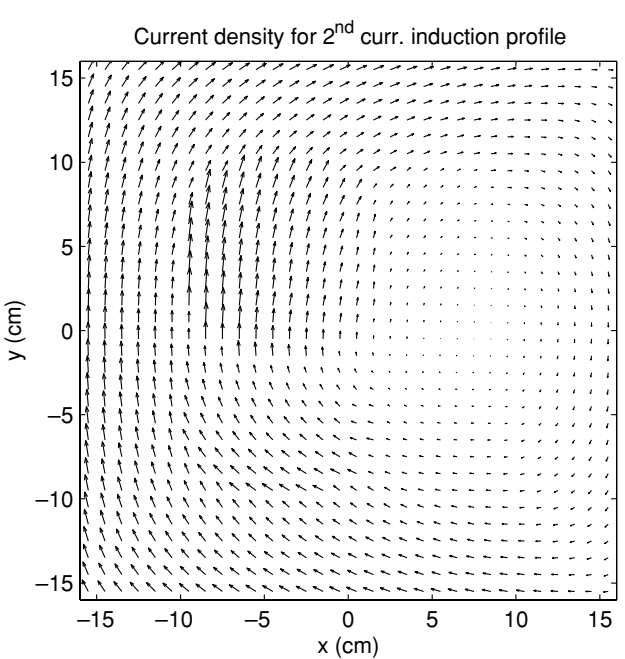

(b)

Figure 11. (a) A vector plot of the current density distribution for slice 32 of the first simulation phantom for the first current induction profile. (b) A vector plot of the current density distribution for slice 32 of the first simulation phantom for the second current induction profile.

absolute conductivity distribution (İder et al 2003, Khang et al 2002). In induced current MR-EIT, since the combined system matrix is not singular, it is possible to find the absolute conductivity distribution inside the object. Therefore, there is no need for any additional voltage measurement in induced current MR-EIT.

For injected current MR-EIT, the need for at least two current injection profiles, even for relative conductivity reconstructions, is explained in Khang et al (2002) and İder et al (2003). With one current injection profile, unique reconstructions are not possible in injected current MR-EIT. In induced current MR-EIT, although the combined system matrix is nonsingular even for one current induction profile, it is nevertheless necessary to use at least two current induction profiles in order to significantly improve its ill-conditioning. Although the uniqueness of our algorithm is not investigated in this study, our proposed induced current MR-EIT method appears to exhibit similar uniqueness characteristics to injected current MREIT.

In figure 11, xy-components of the current densities for two different current induction profiles for the mid-slice (32nd slice) of the first simulation phantom are given. With different 
current induction profiles, current densities at a given location exhibit different directions and this may be the reason for improved condition number of the combined system matrix, using similar arguments as made for injected current MR-EIT (İder et al 2003, Khang et al 2002).

In our simulations, the maximum magnitude of the current density inside the object is calculated to be approximately $7500 \mathrm{~mA} \mathrm{~m}^{-2}$. Lee et al (2003) injected $28 \mathrm{~mA}$ current into a $5 \mathrm{~cm} \times 5 \mathrm{~cm} \times 5 \mathrm{~cm}$ phantom and obtained satisfactory experimental results by using an MRI system with $t_{\text {curr }}=48 \mathrm{~ms}$, and SNR $\simeq 30$. In their study, they used denoising techniques to reduce the effect of noise. In Lee et al (2003), if we assume that the injected current is uniformly distributed inside the object, the current density inside the object is found to be $11200 \mathrm{~mA} \mathrm{~m}^{-2}(28 /(0.05 \times 0.05))$. In Ider and Onart (2004), at least $1000 \mathrm{~mA}$ is used to obtain satisfactory results. The dimensions of the object used in their simulations are the same as our phantom size. With the same assumption as we made for Lee et al's work, the maximum current density inside the object is found to be approximately $4883 \mathrm{~mA} \mathrm{~m}^{-2}$ $(1000 /(0.32 \times 0.64))$. Therefore, in our study, the maximum current density level is of the same order of magnitude as both Lee et al's work and İder and Onart's work. In order to obtain this amount of current density in the object, it is necessary to apply 1000 A current to the external induction coil if the coil is single turn. By using multiple-turn coils this amount of current can be significantly reduced.

Phase accumulating due to $B_{p z}$ is ideally zero. However, in practice, due to $180^{\circ} \mathrm{RF}$ pulse position inaccuracies and because $B_{p z}$ is large $\left(B_{p z}^{\text {peak }}\right.$ is of the order of $\left.5 \mathrm{mT}\right)$, there will be phase accumulating due to $B_{p z}$ as well. Assuming a consistent few $\mu$ s error in pulse position, say $5 \mu \mathrm{s}$, the phase accumulating due to $B_{p z}$ is

$$
\varphi_{P 2}(x, y, z)=2 N(-1)^{N} \gamma B_{p z}^{\text {peak }}(x, y, z) \frac{2 \sin (2 \pi \times 1 \mathrm{kHz} \times 5 \mu \mathrm{s})}{(2 \pi \times 1 \mathrm{kHz})} .
$$

This equation is derived by finding the area under the $\pm 5 \mu$ s region around the peak of the $B_{p z}$ waveform. With $N=39$ and $B_{p z}^{\text {peak }}=5 \mathrm{mT}$ the value of $\varphi_{P 2}$ is $6.6^{\circ}\left(\bmod 360^{\circ}\right)$. In this calculation, phase wrapping is accounted for by $\bmod 360^{\circ}$ division. The phase due to $B_{p z}$ wraps 1042 times. On the other hand, if $B_{p z}$ is inhomogeneous the phase map will be highly noisy due to excessive phase wrapping. For this reason, use of homogenous $B_{p z}$ is recommended in which case the contribution of $B_{p z}$ to the phase will be constant for the whole slice and noisy behavior due to phase wrapping will be eliminated. If $B_{p z}$ is generated by a four coil Helmholtz configuration it is possible to obtain $10 \mathrm{ppm}$ inhomogeneity easily (Lugansky 1987). We have calculated the $B_{p z}$ due to an unoptimized Helmholtz pair which yielded $380 \mathrm{ppm}$ inhomogeneity in the phantom volume (much less on a slice basis, for example only $13 \mathrm{ppm}$ on the mid-slice). This amount of inhomogeneity corresponds to $1.9 \mu \mathrm{T}$. If the above calculation is repeated for $1.9 \mu \mathrm{T}$ without the $\bmod 360^{\circ}$ operation then the inhomogeneity in phase accumulation due to $B_{p z}$ across the volume will be $142.7^{\circ}$. Furthermore, in the imaging equation, equation (29), the Laplacian of $B_{s z}$ is used. Even if $B_{s z}$ is contaminated with the contribution coming from $B_{p z}$, this contamination is further reduced by the Laplacian operation because the Laplacian of $B_{p z}$ is very small. In fact the Laplacian of $B_{p z}$ is theoretically zero due to equation (4). There are however numerical computation errors due to the finite mesh sizes used. To observe the relative contributions of $B_{p z}$ and $B_{s z}$, the Laplacians of $\varphi_{P 2}$ and $\varphi_{S 2}$ are compared in figure 12. The Laplacian of $\varphi_{P 2}$ is six orders of magnitude smaller than the Laplacian of $\varphi_{S 2}$ when the $B_{p z}$ with $1.9 \mu \mathrm{T}$ inhomogeneity is used.

The contribution of $B_{p z}$ to the Larmour frequency at its positive and negative peaks is $\pm 213 \mathrm{kHz}$. Therefore the $180^{\circ} \mathrm{RF}$ pulses must be hard pulses to cover this deviation. However if the frequency of the $180^{\circ} \mathrm{RF}$ pulses is chosen to be $\gamma\left(B_{0} \pm B_{p z}^{\mathrm{peak}}\right), B_{0}$ being the static magnetic field of the MRI system and $B_{p z}^{\text {peak }}$ being the peak value of $B_{p z}$ in time, then 


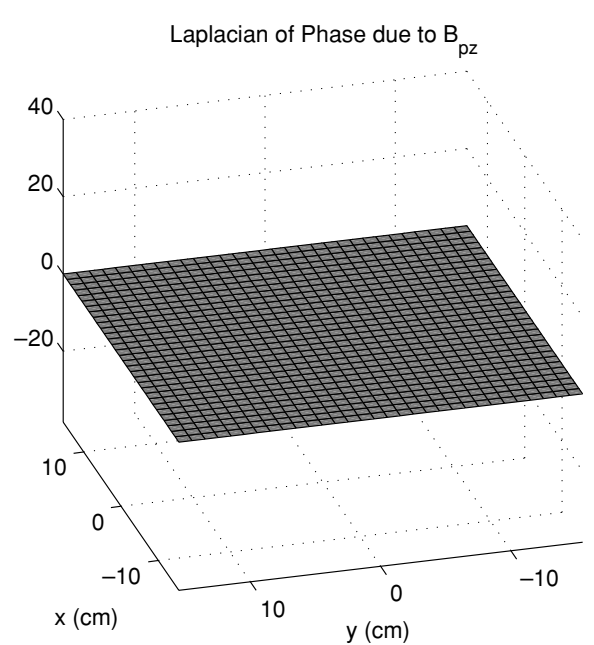

(a)

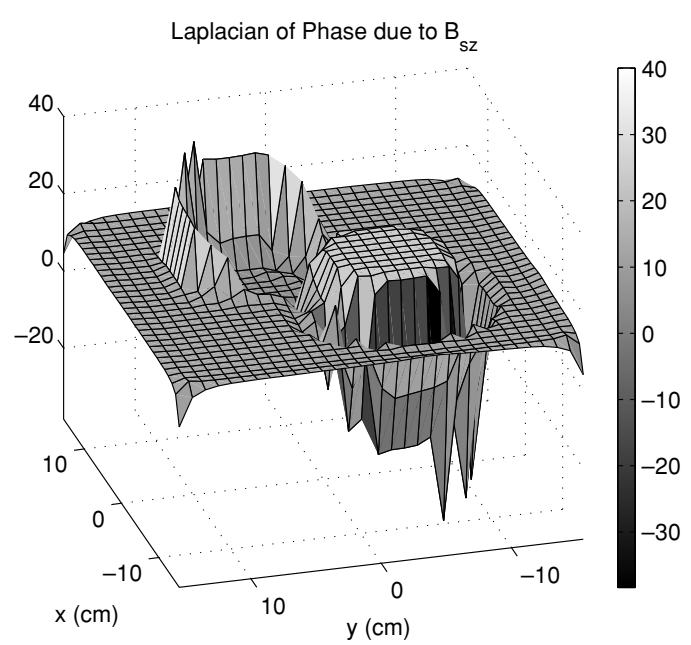

(b)

Figure 12. (a) Laplacian of $\varphi_{P 2}$, the phase accumulating due to $B_{p z}$, (b) Laplacian of $\varphi_{S 2}$, the phase accumulating due to $B_{s z}$. Units are arbitrary.

this problem is removed. Still, it is recommended that the duration of $180^{\circ} \mathrm{RF}$ pulses must be short because during their application $B_{p z}$ is changing. The change in $B_{p z}$ during for example $90 \mu \mathrm{s}$ is $198 \mu \mathrm{T}$ corresponding to a deviation of $8.5 \mathrm{kHz}$. A $100 \mu \mathrm{s}$ hard RF pulse has a frequency range of $11 \mathrm{kHz}$ which covers the $8.5 \mathrm{kHz}$ deviation.

The pulse sequence we have proposed is a modified version of that of Mikac et al (2001). They have applied $39180^{\circ} \mathrm{RF}$ pulses in a period of $20 \mathrm{~ms}$ and have obtained high resolution and high contrast current density images both in phantoms and biological samples. The current density in their experiments with biological samples is $250 \mathrm{~A} \mathrm{~m}^{-2}$. This corresponds to a maximum magnetic field of $1.1 \mu \mathrm{T}$, considering the object geometry they have used. Therefore, when currents are applied into the sample, an additional maximum $1.1 \mu \mathrm{T}$ is imposed on the main magnetic field. The primary magnetic field in our experiments is about $5 \mathrm{mT}$. This value is, of course, much larger than the $1.1 \mu \mathrm{T}$ in Mikac et al's experiments. However, the actual quantity which must be considered is the inhomogeneity in $B_{p z}$, because we can easily choose the $180^{\circ} \mathrm{RF}$ pulses at a center frequency of $\gamma\left(B_{0} \pm B_{p z}^{\text {peak }}\right)$, as explained in the previous paragraph. For the unoptimized Helmholtz pair the inhomogeneity in $B_{p z}$ is $1.9 \mu \mathrm{T}$, similar to $1.1 \mu \mathrm{T}$.

Recently Muftuler et al (2004) have applied a similar pulse sequence for $200 \mathrm{~Hz}$ injected current MR-EIT and have obtained successful images from phantoms and rats. In their pulse sequence $z$-gradients are also applied during the current application time, i.e. during the $180^{\circ}$ pulses. They have used low currents about $4 \mathrm{~mA}$ corresponding to approx. $5700 \mathrm{~mA} \mathrm{~m}^{-2}$.

It is discussed above that homogeneous $B_{p z}$ is advantageous for MRI phase measurements and to decrease errors in the calculation of $\nabla^{2} B_{s z}$. However, the reconstructions made in section 6 are for non-homogeneous $B_{p z}$ distributions which have $76 \%$ inhomogeneity over the whole phantom and $37 \%$ inhomogeneity over the mid-slice. To test whether homogeneous $B_{p z}$ has any disadvantage with respect to image formation, we made reconstruction simulations for the unoptimized Helmholtz pair mentioned above which has $380 \mathrm{ppm}$ inhomogeneity for the first simulation phantom (see figure 13). At the fifth iteration the same image and profile plots as in figure 6 are obtained with the same error values. 


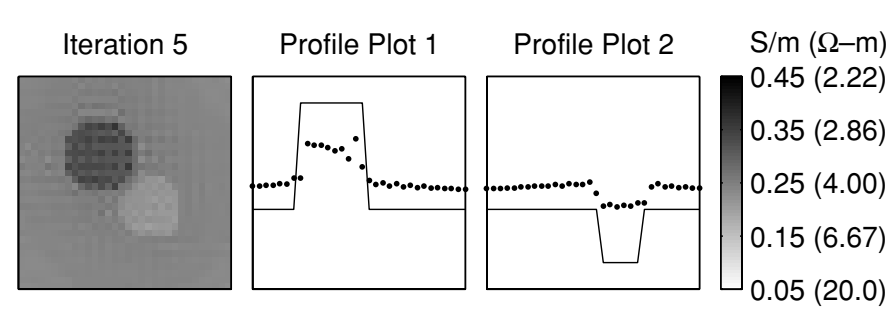

Figure 13. Reconstructed conductivity distribution for the fifth iteration for slice 32 of the first simulation phantom and two profile plots. The description of the profile plots is given in figure 6 .

Another possible tool is the use of signal averaging by repeating the MRI data acquisition several times and averaging the phase images obtained for each. We have not analyzed in this study the possible benefits to be obtained by signal averaging.

Our iterative reconstruction algorithm is not designed to minimize the $L^{2}$ norm of error defined in equation (35). $L^{2}$ error is only used for monitoring the convergence of simulations. In some of our simulations $L^{2}$ error does not always decrease in successive iterations. This issue must be further investigated.

The final reconstruction error is contributed by the errors made in the generation of $B_{s z}$ and $B_{p z}$ data using the forward solver and the errors made in the finite difference approximations given in equations (30) and (31). In figure 12, it can be observed that $\nabla^{2} B_{s z}$ has high values at points and lines where the internal conductivity distribution has high gradients. These are also the regions where the Laplacian calculations yield erroneous values. In order to obtain more accurate results, finer meshes may be utilized. However, using finer meshes will increase the computation time. Our forward solver, which solves the internal eddy current density, takes about 19 min for a current induction profile on a Pentium-IV PC with $2.8 \mathrm{GHz}$ internal clock, 1 GB DDR-RAM and $500 \mathrm{MHz}$ System Bus speed. One iteration of the algorithm takes about 82 min with the same PC. Generating magnetic flux densities, $B_{s z}$ and $B_{p z}$, and magnetic vector potential, $A_{p}$, for four current induction profiles takes about $6 \mathrm{~h}$ using an optimized $\mathrm{C}$-code. These computation times are formidable, and there is need for further work to develop hardware and software solutions to decrease them considerably.

\section{References}

Birgül Ö, Eyüboğlu B M and İder Y Z 2003 Experimental results for 2D magnetic resonance electrical impedance tomography (MR-EIT) using magnetic flux density in one direction Phys. Med. Biol. 48 3485-504

Birgül Ö and İder Y Z 1995 Use of magnetic field generated by the internal distribution of the injected currents for electrical impedance tomography Proc. 9th Int. Conf. on Bio-Impedance pp 418-9

Gençer N G, Kuzuo ğlu M and İder Y Z 1994 Electrical impedance tomography using induced currents IEEE Trans. Med. Imaging 13 338-50

İder Y Z and Birgül Ö 1998 Use of magnetic field generated by the internal distribution of injected currents for electrical impedance tomography (MR-EIT) Elektr. Turk. J. Elec. Eng. Comput. Sci. 6 591-604

İder Y Z and Onart S 2004 Algebraic reconstruction for 3D magnetic resonance-electrical impedance tomography (MREIT) using one component of magnetic flux density Physiol. Meas. 25 281-94

İder Y Z, Onart S and Lionheart W R B 2003 Uniqueness and reconstruction in magnetic resonance electrical impedance tomography (MR-EIT) Physiol. Meas. 24 591-604

Khang H S, Lee B I, Oh S H, Woo E J, Lee S Y, Cho M H, Kwon O, Yoon J R and Seo J K 2002 J-substitution algorithm in magnetic resonance electrical impedance tomography (MR-EIT): phantom experiments for static resistivity images IEEE Trans. Med. Imaging 21 695-702

Kwon O, Woo E J, Yoon J R and Seo J K 2002 Magnetic resonance electrical impedance tomography (MR-EIT): simulation study of j-substitution algorithm IEEE Trans. Biomed. Eng. 49 160-7 
Lee B I, Oh S H, Woo E J, Lee S Y, Cho M H, Kwon O, Seo J K and Baek W S 2003 Static resistivity image of a cubic saline phantom in magnetic resonance electrical impedance tomography (MREIT) Physiol. Meas. 24 579-89

Lugansky L B 1987 Optimal coils for producing uniform magnetic fields J. Phys. E: Sci. Instrum. 20 277-85

Mikac U, Demšar F, Beravs K and Serša I 2001 Magnetic resonance imaging of alternating electric currents Mag. Reson. Imaging 19 845-56

Muftuler L T, Hamamura M, Birgul O and Nalcioglu O 2004 Resolution and contrast in magnetic resonance electrical impedance tomography (MREIT) and its application to cancer imaging Technol. Cancer Res. Treat. $3599-609$

Oh S H, Lee B I, Woo E J, Lee S Y, Cho M H, Kwon O and Seo J K 2003 Conductivity and current density image reconstruction using harmonic $B_{z}$ algorithm in magnetic resonance electrical impedance tomography $P h y s$. Med. Biol. 48 3101-16

Schnell W, Renz W, Vester M and Ermert H 2000 Ultimate signal-to-noise-ratio of surface and body antennas for magnetic resonance imaging IEEE Trans. Antennas Propag. 48 418-28

Scott G C, Joy L G, Armstrong R L and Henkelman R M 1992 Sensitivity of magnetic-resonance current density imaging J. Mag. Res. 97 235-54

Seo J K, Kwon O, Lee B I and Woo E J 2003a Reconstruction of current density distributions in axially symmetric cylindrical sections using only one component of magnetic flux density: computer simulation study Physiol. Meas. 24 565-77

Seo J K, Yoon J R, Woo E J and Kwon O 2003b Reconstruction of conductivity and current density images using only one component of magnetic field measurements IEEE Trans. Biomed. Eng. 50 1121-4

Silvester P P and Ferrari R L 1996 Finite Elements for Electrical Engineers (Cambridge: Cambridge University Press) 